\title{
8 Digitalisierung im Rettungsdienst
}

\author{
Thomas Luiz
}

Die Digitalisierung hat in allen Teilbereichen des Rettungsdienstes zugenommen - infolge der föderalen Struktur des Rettungsdienstes und unterschiedlicher Finanzierungsvoraussetzungen allerdings lokal in unterschiedlichem Ausmaß. Die bestehende Vielzahl an Hard- und Softwareumgebungen sowie unterschiedliche organisatorische und rechtliche Rahmenbedingungen erschweren die für eine gut funktionierende medizinische Versorgungskette notwendige Vernetzung von Leitstellen, Rettungsdiensten und Kliniken. Großes Potenzial zur Optimierung der Notfallversorgung liegt in der telemedizinischen Unterstützung nichtarztbesetzter Rettungsmittel und in der Nutzung von Echtzeitdatenbanken, welche aufnahmebereite Kliniken anzeigen. Bestehende Defizite betreffen v.a. das Fehlen strukturierter Notrufabfragesysteme in Leitstellen, die Beschränkung von Notrufen auf Sprachtelefonie sowie Lücken in der Mobilfunkabdeckung im ländlichen Bereich. Die gewachsene Komplexität der eingesetzten IT-Systeme und die zunehmende Abhängigkeit von IT-gestützten Prozessen beinhalten jedoch auch Risiken, gegen die sich die Betreiber absichern müssen. Ein wichtiger Erfolgsfaktor bei der digitalen Transformation liegt darin, die Beteiligten offen zu informieren und umfassend zu schulen. KI-basierte Anwendungen werden mittelfristig Einzug in den Rettungsdienst halten und die Arbeitsumgebung maßgeblich beeinflussen, v.a. in den Leitstellen (s. Abb. 1). 


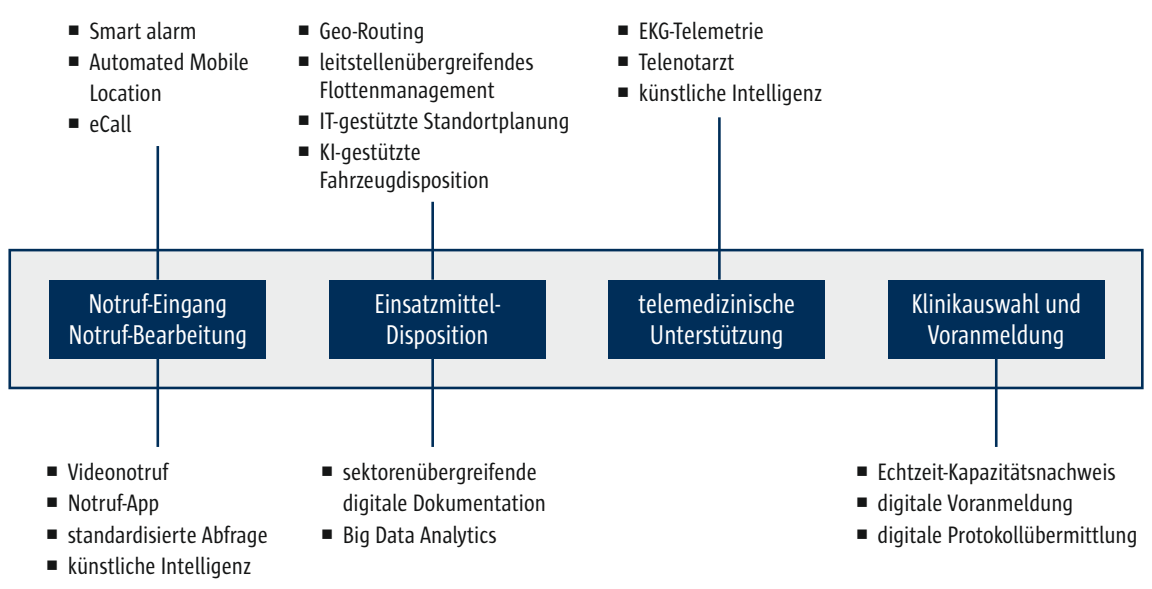

Abb. 1 Überblick: Prozessunterstützung im Rettungsdienst durch digitale Technologien

\subsection{Einführung}

2017 erfolgten laut Statistischem Bundesamt in Deutschland ca. 16,3 Millionen Einsatzfahrten im Rettungsdienst, davon ca. 60\% Notfall- und Notarzteinsätze. Statistisch nimmt damit jeder Fünfte einmal jährlich den Rettungsdienst in Anspruch. Damit ist der Rettungsdienst ein zentraler Baustein im System der Gesundheitsversorgung und Gefahrenabwehr.

Im Eckpunktepapier der medizinischen Fachgesellschaften zur notfallmedizinischen Versorgung wird die Notwendigkeit einer optimal organisierten Notfallversorgung für 6 medizinisch und volkswirtschaftlich besonders wichtige „Tracerdiagnosen“ (ST-Hebungs-Myokardinfarkt, Schlaganfall, Kreislaufstillstand, Sepsis, Polytrauma und Schädel-Hirn-Trauma) herausgestellt (Fischer 2016). Die in diesem Whitepaper gestellten Forderungen umfassen den gesamten Versorgungsprozess („Rettungskette“) vom Notruf bis zur Klinik und definieren den Rahmen, an welchem die Strukturen und Prozesse ausgerichtet sein sollten. Zentrale Punkte des Konzepts sind Zeitfenster, innerhalb derer die verschiedenen Teilprozesse abgeschlossen sein sollen (s. Abb. 2), die Zuweisung in eine geeignete Klinik sowie ein an diesen Erfordernissen ausgerichteter strukturierter Informationsaustausch zwischen den Beteiligten. Beide Faktoren, kurze Versorgungszeiten und die Behandlung in einer geeigneten Zielklinik, verbessern bei den genannten Diagnosen nachweislich die Prognose (Fischer 2016). 


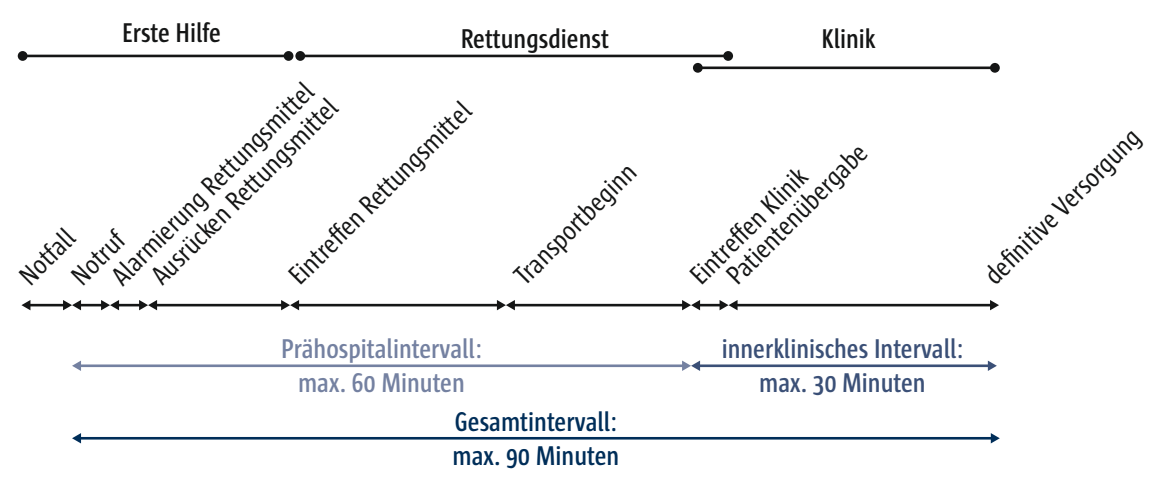

Abb. 2 Zeitintervalle gemäß Eckpunktepapier 2016 zur notfallmedizinischen Versorgung der Bevölkerung in Präklinik und Klinik

\section{Das Eckpunktepapier - Wie steht es um die Umsetzung?}

Im Alltag werden die Forderungen des Eckpunktepapiers häufig nicht eingehalten. So beträgt beim Polytrauma das mittlere Intervall bis zur definitiven Versorgung in Deutschland anstelle der geforderten max. 90 Minuten mehr als 120 Minuten (Höfer u. Lefering 2018). In BadenWürttemberg werden mehr als 20\% der Polytraumatisierten nicht in eine geeignete Klinik transportiert, und beim Schlaganfall und beim Myokardinfarkt wird das angestrebte Prähospitalintervall von max. 60 Minuten bei 25\% der Patienten nicht eingehalten (SQR BW 2018). Die Praxis zeigt, dass dies auch an fehlender IT-Unterstützung liegt.

Vor diesem Hintergrund liegt es nahe, die Versorgung mittels moderner Informationstechnologie (IT) zu unterstützen und zu beschleunigen. So sehen die Planungen des Bundesgesundheitsministeriums zur Integrierten Notfallversorgung die elektronische Vernetzung von Leitstellen, Rettungsdienst und Notaufnahmen vor (Bundesgesundheitsministerium 2019). Auch der Deutsche Städtetag hat kürzlich die Kommunen dazu aufgefordert, sich auf die Digitalisierung und intelligente Vernetzung im Rettungsdienst vorzubereiten (Deutscher Städtetag 2019).

Der Beitrag will zeigen, welchen Beitrag digitale Technologien im Rettungsdienst leisten können und wie die Nutzung in Deutschland und anderen europäischen Ländern vorangeschritten ist. Sofern die Daten frei zugänglich waren, wird der konkrete Durchdringungsgrad dargestellt. Bei zahlreichen Aspekten kann dies mangels offizieller Statistiken allerdings nur geschätzt werden. 


\subsection{Notfalldetektion und -meldung}

\subsubsection{Mobiltelefonie}

Die Nutzung der Mobilfunktechnologie ist für die meisten Menschen selbstverständlicher Teil ihres Alltags. So nutzten 2019 in Deutschland ca. 58 Mio. Menschen ein Smartphone. Bei den unter 50-Jährigen waren es 2017 mehr als $95 \%$, und bei den mindestens 65 -Jährigen 41\%. EU-weit wurden 2019 mehr als $70 \%$ der Notrufe über Mobiltelefone abgegeben. In Deutschland waren es $64 \%$, in Finnland sogar über 95\% (European Commission 2020).

Es liegt für die Bürger nahe, zentrale Funktionalitäten dieser Technologie wie Standortübermittlung oder Videotelefonie auch bei Notrufen zu nutzen. Bislang sind Notrufe an die Leitstellen jedoch noch weitestgehend auf die klassische Sprachtelefonie (Notruf 112) begrenzt. Allerdings fordert die Richtlinie des Europäischen Parlaments über den Europäischen Kodex für die elektronische Kommunikation (EECC), welche bis Ende 2020 umgesetzt werden muss, dass Notrufe nicht nur als Sprachanrufe, sondern auch per Videoverbindung und weiteren Kommunikationsarten kostenfrei verfügbar sein müssen. Zwar haben alle Bundesländer der Einführung einer bundeseinheitlichen Notruf-App zugestimmt. Es ist allerdings fraglich, ob diese bis Ende 2020 bundesweit verfügbar sein wird. Es obliegt daher aktuell der individuellen Entscheidung von Leitstellen, überbrückend Messenger-Dienste zu nutzen teils in einer rechtlichen Grauzone bezüglich des Datenschutzes. Genauere Daten zum Verbreitungsgrad liegen hierfür allerdings nicht vor.

Das enorme Potenzial von Smartphones bei der Übermittlung von Notrufen wird aktuell noch unzureichend genutzt.

Im Gegensatz zu Notrufen über Festnetzanschlüsse, bei welchen der Standort des Telefons automatisch angezeigt wird, mussten die Leitstellenmitarbeiter bei Notrufen über Mobiltelefone die aktuelle Position der Anrufer bislang mündlich erfragen. Dieser zeitraubende Vorgang war Anlass für die Entwicklung von Apps, welche der Leitstelle die Geoposition des Anrufers mittels GPS (Global Positioning System)-Modul sowie ggf. weitere Informationen automatisch übermitteln. Manche dieser Apps sind nur regional verfügbar oder kostenpflichtig, andere kostenlos und weltweit verfügbar.

Mit der Einführung des Automated Mobile Location (AML)-Verfahrens erfolgt die Standortübermittlung beim Absetzen des Notrufs 112 über Smartphones zukünftig vollautomatisch. Anfang 2020 waren bereits mehrere Dutzend Leitstellen in Deutschland zum Empfang dieser Daten ertüchtigt - der Großteil der Leitstellen sollte innerhalb weniger Monate folgen. Andere Länder, u.a. Belgien, Großbritannien, und die Niederlande, haben AML schon vor Jahren eingeführt. 


\subsubsection{Automatische Notfallerkennung}

Telefonische Hausnotrufsysteme sind in Deutschland zwar weit verbreitet, ihr Nutzen bei Notfällen ist allerdings nur unzureichend untersucht. Grundsätzlich stoßen diese Systeme in Situationen, in denen die Patienten nicht mehr in der Lage sind, aktiv einen Alarm auszulösen, an ihre Grenzen (z.B. Kreislaufstillstand, Krampfanfall, Lähmung). Umgekehrt zeigt die Praxis, dass viele Hausnotrufeinsätze des Rettungsdienstes medizinisch nicht indiziert sind. Die „klassischen“ Systeme mit Druckknopfauslösung einer Telefonverbindung werden daher zunehmend um neue Funktionen wie z.B. die automatische Detektion von Stürzen und Hilflosigkeit, oder die Erkennung von Auffälligkeiten in den Alltagsaktivitäten erweitert. Diese sog. Ambient Assisted Living (AAL)-Lösungen sollen sowohl eine bessere Detektion akuter Notfälle als auch eine Frühwarnung bei allmählicher Verschlechterung des Gesundheitszustands ermöglichen (Gebert et al. 2015). Die Technologie stellt dabei einen Baustein in einem umfassenderen medizinischen und sozialen Betreuungsansatz dar. Eine technische Herausforderung ist die automatische Situationsbewertung in Haushalten mit mehr als einem Bewohner, bei Menschen mit größeren Haustieren sowie bei Menschen mit sich häufig änderndem Tagesablauf. Zudem ist AAL auf die Wohnung begrenzt, erfordert technische Installationen und ist mit beträchtlichen Kosten verbunden. Die Realisierung ist deshalb bislang auf Einzelprojekte beschränkt.

Ein anderer Ansatz für die automatische Notfallerkennung liegt in „Smart Wearables“. Derzeit nutzen 36\% in Deutschland bereits eine Smart Watch. Ein Großteil dieser Geräte misst auch notfallmedizinisch relevante Parameter wie Pulsfrequenz oder den Herzrhythmus. Teilweise besteht bereits eine Zulassung als Medizinprodukt zur Detektion von Vorhofflimmern. Weitere Funktionen, wie die Blutdruckmessung, stehen kurz vor der Einführung.

Eine automatische Notrufauslösung an Leitstellen des Rettungsdienstes ist derzeit jedoch weder mittels AAL-Technologie, noch mittels Smart Wearables möglich. Die Gründe hierfür liegen sowohl in den rechtlichen Rahmenbedingungen, den hohen Anforderungen an die zuverlässige Erkennung (Messgenauigkeit, Sensitivität und Spezifität von Alarmen) als auch in den fehlenden Standards zur Datenübermittlung.

Bereits realisiert ist hingegen die automatische Meldung von Verkehrsunfällen mittels des sog. eCall. Bei diesem System, das seit 2018 in der EU bei neu zugelassenen PKW-Modellen zur Pflichtausstattung zählt, erfassen Fahrzeugsensoren extreme negative Beschleunigungswerte, wie sie bei einem Aufprall auftreten, und senden über ein fest eingebautes Mobilfunkmodul die Fahrzeugposition und -fahrtrichtung sowie Typ und Modell des Unfallfahrzeugs an die Leitstelle. Parallel wird eine Sprachverbindung aufgebaut. Die Erfahrungen mit dem System sind noch sehr begrenzt. 2019 wurden EU-weit erst einige tausend eCalls abgesetzt, gegenüber ca. 158 Millionen Notrufen an die Nummer 112. 


\section{Automatisierte Notfallerkennung und -meldung}

Die automatische Detektion und Meldung von Notfällen ist aus notfallmedizinischer Sicht eine entscheidende Komponente zur Senkung des therapiefreien Intervalls bei lebensbedrohlichen Erkrankungen und Verletzungen. Wie mühsam die Umsetzung solch wegweisender Ideen ist, zeigt die Historie des eCall und der AAL-Forschung. Smart watch-basierte Lösungen könnten ein wesentlich kostengünstigerer und schneller zu realisierender Weg sein, wenn herstellerseitig eine Übereinkunft zu technischen Standards und Schnitttstellen gelingt.

\subsection{Strukturierte Notrufabfrage}

\subsubsection{Hintergrund}

Integrierte Leitstellen sind die Anlaufpunkte für eine enorme Anzahl und Bandbreite an Hilfeersuchen; in Leitstellen mit einem Einzugsbereich von ca. 1 Million Menschen sind es jährlich über 50o.ooo Anrufe. Die Anlässe reichen von der Frage nach diensthabenden Ärzten über Fragen zum Verhalten bei banalen Krankheitssymptomen bis zum Melden eines Kreislaufstillstands oder eines Massenanfalls von Verletzten (MANV). Ebenso heterogen wie die Anlässe für Anrufe sind die in der Leitstelle erfolgenden Reaktionen. Neben der Entsendung von Rettungsmitteln umfassen diese das bloße Mitteilen von Telefonnummern, das Erteilen von Erste Hilfe-Hinweisen oder die Weiterleitung von Anrufern an den Hausarzt, Ärztlichen Bereitschaftsdienst sowie eine Vielzahl weiterer medizinischer und sozialer Dienstleister. Dabei befinden sich die Leitstellen permanent in dem Spannungsfeld, einerseits rasch und sicher ausgefallene Vitalfunktionen zu erkennen, zugleich jedoch die nur sehr begrenzt zur Verfügung stehenden Notfallrettungsmittel nicht „leichtfertig“ zu disponieren. Angesichts der großen Anzahl und Bandbreite an Anrufen, der Bedeutung der raschen Auswahl des richtigen „Effektors“ und einer angemessenen Beratung der Anrufer liegt nahe, diese Prozesse so weit wie möglich zu standardisieren und durch IT zu unterstützen.

\subsubsection{Erfahrungen mit Strukturierten Abfragesystemen}

Unabhängig von der Art der Abfrage („frei“ versus „strukturiert“ bzw. „standardisiert") besteht die zentrale Aufgabe darin, die im Anruf ermittelten Informationen hinsichtlich Akuität und Gefährdungsgrad zu bewerten. Dies mündet in der Regel in einem oder mehreren Einsatzstichworten, welche wiederum Grundlage der Einsatzmitteldisposition sind (s.u.). Je nach Situation werden den Anrufern auch Verhaltenshinweise gegeben. 


\section{Eine qualitativ hochwertige Notrufabfrage ist die entscheidende Voraus- setzung für die Effektivität und Effizienz des Rettungsdienstes.}

In den meisten Nationen mit einem hochentwickelten Gesundheitssystem werden Notrufe IT-unterstützt standardisiert abgefragt, und es liegen viele Publikationen vor, welche die Eignung dieser Systeme hinsichtlich der Einschätzung des zugrundeliegenden Notfalls untersuchten. Bei der Interpretation der Studien ist zu beachten, dass sie nicht nur von Struktur und Inhalt der Abfragealgorithmen beeinflusst werden, sondern auch von weiteren Faktoren, wie Zugang der Bevölkerung zum rettungsdienstlichen Notruf, parallel bestehenden weiteren Notrufnummern, Struktur der Anrufer, Ausbildung des Leitstellenpersonals, Personalstärke der Leitstellen, Ausbildungsniveau des Rettungsdienstes sowie der Vollständigkeit und Genauigkeit der zur Analyse herangezogenen medizinischen Dokumentation. Bei notfallmedizinischen Tracerdiagnosen zeigen die etablierten IT-basierten Abfragesysteme durchaus Schwächen. So betrug beispielsweise die Sensitivität zur Detektion eines Schlaganfalls max. 58\%, und der positiv prädiktive Wert max. 49\% (Buck et al. 2009; Deakin et al. 2009; Ellensen et al. 2018). Untersuchungen zum Kreislaufstillstand aus Großbritannien zeigen eine Sensitivität von $76 \%$ und einen positiv prädiktiven Wert von nur 27\% (Deakin et al. 2017). Die Ergebnisse beim Kreislaufstillstand zeigen, dass zugunsten eines besseren Erkennens dieses zeitkritischsten aller Notfälle eine relativ hohe Rate falsch positiver Einschätzungen in Kauf genommen werden muss. Aufgrund der nahezu weltweiten routinemäßigen Verwendung von Abfragealgorithmen liegen nur wenige aktuelle Daten zur Treffsicherheit der Notrufabfrage ohne Nutzung eines strukturierten Abfrageprozesses vor. Eine ältere Untersuchung aus dem Londoner Rettungsdienst zeigte, dass sich die Sensitivität der Erkennung eines prähospitalen Kreislaufstillstands nach Einführung eines standardisierten Abfragesystems von $15 \%$ auf 50\% mehr als verdreifachte (Heward et al. 2004). Eine ähnliche geringe Sensitivität einer unstrukturierten Notrufabfrage wurde in einer neueren Studie aus Triest, Italien, deutlich, in welcher die Leitstelle lediglich bei 14\% der reanimationspflichtigen Patienten einen Kreislaufstillstand erkannt hatte (Sanson et al. 2016).

In Deutschland werden strukturierte IT-gestützte Abfragesysteme in deutlich geringerem Maße genutzt. Amtliche Statistiken fehlen jedoch. Einer Befragung aus dem Jahr 2017 zufolge, an der sich 100 der geschätzt ca. 220 Leitstellen beteiligten, lag der Implementierungsgrad noch unter 50\% (Luiz et al. 2019). Aufgrund der subjektiven Einschätzung verbesserte sich die Abfrage- und Dispositionsqualität bei mehr als 9o\% der teilnehmenden Leitstellen. Objektive Qualitätsparameter erhoben jedoch nur 41-73\% der Leitstellen. Danach verkürzte sich bei $55 \%$ die Notrufannahmezeit, bei $40 \%$ blieb sie weitgehend unverändert, und nur bei 5\% stieg sie um 10-20\% an. Die Notrufabfragezeit 
verkürzte sich bei ca. 32\% der Leitstellen, blieb bei ca. 36\% weitgehend unverändert und verlängerte sich bei weiteren $32 \%$. Eine Verlängerung der Abfragezeit ist nicht a priori negativ zu beurteilen. Entscheidend ist vielmehr, wie diese zusätzliche Zeitspanne die Qualität der Einschätzung des Meldebilds beeinflusst. Hierbei liegen für den Kreislaufstillstand Hinweise vor, dass bei zu schnellen unsystematischen Abfragen Fehleinschätzungen im Sinne einer tödlichen Untertriage drohen (Palma et al. 2014). Arztbesetzte Rettungsmittel sind die knappsten Ressourcen der Leitstelle. In der o.g. Studie (Luiz et al. 2019) blieb der Anteil der Notarzteinsätze an den Notfalleinsätzen in $46 \%$ der Fälle weitgehend unverändert, verringerte sich bei $43 \%$ leicht und stieg bei $11 \%$ leicht an. Auch die Notarztnachalarmierungsquote blieb in $48 \%$ der Fälle unverändert, verminderte sich bei weiteren $48 \%$ und erhöhte sich nur bei $4 \%$. Eine ältere Untersuchung aus Berlin zeigte eine niedrigere Notarztquote, wenn Notrufe strukturiert abgefragt wurden, im Vergleich zur freien Abfrage (Baumann et al. 2009). Die Befürchtung, dass Notrufabfragesysteme einen Anstieg der Notarzteinsätze zur Folge hätten, lässt sich anhand jener Untersuchungen somit nicht bestätigen. Gleichzeitig zeigte eine weitere Berliner Studie zu Patienten mit einem Kreislaufstillstand, dass die entscheidenden Parameter Bewusstsein und Atmung bei freier Abfrage signifikant schlechter und seltener abgefragt wurden, als bei Nutzung eines Abfrageprotokolls (Sellin 2011). Angesichts der Bedeutung der Notrufabfrage ist bedauerlich, dass keine weiterführenden Untersuchungen aus deutschen Leitstellen vorliegen.

\begin{abstract}
Notrufabfrage
Aus Deutschland liegen bislang nur wenige Erkenntnisse zur Qualität der Notrufabfrage vor. Analysiert wird vor allem die Dauer von Teilprozessen, ohne dass bislang ein klarer Bezug zur erzielten Qualität besteht. Die Nutzung IT-gestützter standardisierter bzw. strukturierter Abfragesysteme ist im Gegensatz zu vielen anderen Nationen noch kein Standard.
\end{abstract}

\title{
8.3.3 Telefonische Unterstützung der Anrufer
}

Die telefonische Anleitung von Anrufern zu Erste Hilfe-Maßnahmen ist eine Kernforderung der Fachgesellschaften. Dies gilt v.a. für den Kreislaufstillstand (sog. dispatcher-assisted CPR, oder Telefon-CPR, T-CPR) (Fischer et al. 2016), da diese Maßnahme geeignet ist, die Überlebensrate etwa zu verdoppeln (Böttiger et al. 2020). Einer europäischen Multicenter-Studie zufolge wurde im Jahr 2017 bei 53\% der Kreislaufstillstände eine T-CPR durchgeführt (Gräsner et al. 2020). In Deutschland waren es den Daten des Deutschen Reanimationsregisters zufolge dagegen nur 20,9\% (Fischer et al. 2018). Einschränkend ist anzumerken, dass in beiden Studien lediglich etwas mehr als ein Drittel der Gesamtbevölkerung erfasst wurde. 
Leitstellen können durch telefonische Anleitung der Anrufer das Überleben von Patienten mit einem Kreislaufstillstand erheblich steigern.

Software-Tools können Leitstellenmitarbeiter bei der telefonischen Anleitung zu Erste Hilfe-Maßnahmen sehr wirksam unterstützen und dazu beitragen, die Rate an T-CPR zu erhöhen. Konkrete Hilfen beinhalten z.B. auf das Alter der Anrufer und der Patienten zugeschnittene Texthinweise zur Durchführung der Reanimation, elektronische Metronome, die visuelle Anzeige der geleisteten Thoraxkompressionen bzw. Beatmungen u.a.m. Diese Unterstützung nehmen die Mitarbeiter in den Leitstellen gut an: So erhöhte sich in der o.g. Befragung die Rate an T-CPR nach Einführung der Strukturierten Notrufabfrage bei 94\% der teilnehmenden Leitstellen (Luiz et al. 2019). Auch in anderen Studien wurde die T-CPR bei Nutzung entsprechender IT-basierter Algorithmen signifikant häufiger eingesetzt (Sellin 2011; Stipulante et al. 2014; Utstein Osaka Project 2015).

\subsubsection{Zusammenfassung}

Strukturierte Notrufabfragesysteme sind in Deutschland noch keineswegs Standard. Daten zur Prozess- und Ergebnisqualität liegen bislang nur in sehr begrenztem Maße vor. Sie deuten jedoch an, dass diese Systeme signifikant zur Verbesserung der Abfragequalität und der Unterstützung der Anrufer beitragen können. Die Sensitivität und die Spezifität der bisher genutzten Abfragesysteme sind begrenzt, da die verwendeten Algorithmen weitgehend „statisch“ arbeiten und Optimierungen eine aufwändige Zusammenführung der Leitstellendaten mit den medizinischen Daten des Rettungsdienstes erfordern. Dies legt nahe, die bestehenden Algorithmen durch innovative Ansätze der Situationsbewertung zu ergänzen (s.u.).

\subsection{Situationsbewertung}

Da eine Beurteilung des Patientenzustands allein anhand mündlich übermittelter Informationen auch bei Nutzung standardisierter Abfragealgorithmen nur begrenzt möglich ist, werden große Hoffnungen in Videonotrufe gesetzt. Neben den bekannten Social-Messenger oder allgemeinen Videotelefonie-Diensten sind mittlerweile auch spezifische kostenpflichtige Lösungen für Leitstellen verfügbar. Besonders im Fokus steht die Videoübertragung bei bewusstlosen Patienten. Dort soll sie dazu beitragen, einen Kreislaufstillstand rascher zu erkennen, die Anleitung von Ersthelfern zu einer Reanimation zu verbessern und damit die Prognose von Reanimationspatienten zu optimieren. Die wenigen bislang vorliegenden Studien erlauben dazu jedoch noch keine klare Aussage (Soar et al. 2019; Lee et al. 2020). 


\section{Videonotrufe und Verfahren der Künstlichen Intelligenz sind aktuell in Leitstellen erst vereinzelt implementiert.}

Ein anderer Ansatz beinhaltet den Einsatz von Methoden der Künstlichen Intelligenz (KI). Dies beinhaltet z.B. eine automatische Identifikation von Schlüsselwörtern im laufenden Telefonat (z.B. „grau im Gesicht“) sowie die zusammenfassende automatische Bewertung einer Kombination von gefallenen Begriffen. Eine erste Untersuchung aus Dänemark zeigte, dass ein KI-basierter Algorithmus bei Patienten mit einem Kreislaufstillstand einer konventionellen standardisierten Notrufabfrage sowohl hinsichtlich der Sensitivität, als auch der Schnelligkeit der Symptomeinordnung leicht überlegen war (Blomberg et al. 2019). Ein wichtiges Ziel bei der Entwicklung solcher Lösungen ist, die KI fortlaufend zu „trainieren “ und ihre Treffergenauigkeit stetig zu optimieren (,deep learning“). Dazu müssen die von der KI getroffenen Bewertungen mit den in Einsatzprotokollen des Rettungsdienstes beschriebenen Diagnosen validiert werden. Ein weiterer Ansatz zur Optimierung der „Trefferquote" ist, die im Notruf erhobenen Informationen mit weiteren Datenquellen (z.B. Tageszeit, Anrufercharakteristika, Wetter, Quelle des Notrufs, etc.) zu verknüpfen.

\subsection{Standortplanung}

Die Entscheidungen bezüglich des Standorts wurden für einen Großteil der bestehenden Wachen bereits vor Jahrzehnten getroffen. Die damaligen Entscheidungsgrundlagen sind heute häufig nicht mehr bekannt oder teilweise durch geänderte Rahmenbedingungen (z.B. neue Verkehrstrassen, Neubaugebiete, Schließung von Klinikstandorten etc.) sogar obsolet. Das Spannungsfeld von hohem Kostendruck, steigenden Einsatzzahlen und zunehmenden Eintreffzeiten der Rettungsmittel sowie der notwendigen Rechtssicherheit und Transparenz bei der Festlegung neuer Wachenstandorte lässt sich letztlich nur mittels mathematischer Modellierung befriedigend lösen. Entsprechende Lösungsansätze wurden erstmals vor ca. 15 Jahren vorgestellt (Peleg u . Pliskin 2004). Daten, die in derartige mathematische Modelle fließen, sind v.a. Anzahl, Typ und Dienstzeiten der Rettungsmittel der zu untersuchenden Wache bzw. Wachen, örtliche Verteilung von Kliniken, Einwohnerzahl der versorgten Gebiete sowie die zeitliche Verteilung und Dauer früherer Einsätze zu definierender Wachen bzw. Regionen - letztere kann im Extremfall ein ganzes Bundesland umfassen.

Nur die Standortplanung auf Basis mathematischer Modelle bietet die notwendige objektive Entscheidungsgrundlage. 
Auf dieser Grundlage werden dann Simulationen durchgeführt, die für eine oder mehrere Wachen oder sogar für ein ganzes Bundesland die aus mathematischer Sicht optimalen Standorte und Anzahl an Rettungsmitteln errechnet. Dabei können z.B. die Auswirkungen des Verschiebens, Streichens oder Hinzufügens von Wachen auf die Eintreffzeiten bzw. Erreichbarkeit von Gemeinden konkret betrachtet und Diskussionen über Standortfragen neutraler geführt werden. Wie viele Standorte mittlerweile auf einer solchen Crundlage überprüft bzw. neu geplant werden, ist nicht bekannt, da einschlägige Gutachten und Verhandlungsergebnisse häufig nicht öffentlich einsehbar sind. Eine Ausnahme bildet Bayern, wo seit Ende der neunziger Jahre in regelmäßigem Abstand die Standorte aller Rettungswachen überprüft und optimiert werden. Zumindest die ersten beiden dieser sog. TRUST-Gutachten sind bis heute im Internet frei downloadbar (INM 2005).

\subsection{Disposition der Rettungsmittel}

Die Umsetzung der in der Notrufabfrage ermittelten Informationen in eine konkrete Alarmierung von Einsatzmitteln ist nur durch die simultane Betrachtung der taktischen Lage möglich (v.a. aktueller Status und Position der Rettungsmittel). Aufgrund der Komplexität dieses Sachverhalts erfolgt dies softwaregestützt mittels des Einsatzleitsystems, ELS. Im Zuge der Ausrüstung der Fahrzeuge mit GPS und Datenfunkgeräten, welche ihre aktuelle Position an die Leitstelle übermitteln, begannen die ersten Leitstellen vor ca. 10-12 Jahren damit, Einsatzmittel dynamisch zu disponieren. Dabei wird zur Verkürzung der Eintreffzeit nicht die dem Einsatzort nächstgelegene Wache, sondern das aktuell am nächsten stehende, einsatzbereite Fahrzeug alarmiert. Es ist allerdings für die Erzielung optimaler Ergebnisse wichtig, die im sog. Georouting ermittelten Einsatzmittelvorschläge und die realen Fahrzeiten in regelmäßigen Abständen zu überprüfen und ggf. die dem Routing zugrundeliegenden Parameter, z.B. Durchschnittsgeschwindigkeit von Rettungsmitteltypen, anzupassen. Mittlerweile dürfte der allergrößte Teil der Leitstellen in Deutschland Georouting praktizieren. In vielen europäischen Ländern ist das Georouting ebenfalls verfügbar. Eine steigende Zahl von Leitstellen ist mittlerweile elektronisch mit Nachbarleitstellen vernetzt. Diese elektronische Einsatzübergabe erübrigt v.a. bei Einsätzen in grenznahen Bereichen oder bei einem MANV zeitraubende telefonische Anfragen. Technisch gibt es keine Gründe, eine solche Vernetzung auf die Grenzen von Bundesländern zu beschränken, lediglich politische.

In einigen Regionen (z.B. Hessen) ist es Standard, eine infolge eines Einsatzes „leere“ Wache temporär durch Verlegen eines Fahrzeugs der Nachbarwache zu besetzen oder das Fahrzeug der Nachbarwache temporär an der Grenze der beiden Gebiete zu positionieren. Ziel ist es, bei einem weiteren Notfall die Anfahrtszeit zu verkürzen. Diese Vorgehensweise ist nicht notwendigerweise immer auch die bestmögliche, da die Fähigkeit der Disponenten, die taktische 
Lage korrekt zu beurteilen, interindividuell variiert und v.a. in großen Leitstellenbereichen oder zu Zeiten einer hohen Einsatzbelastung an Grenzen stößt.

Eine andere Herangehensweise ist daher, auf der Datenbasis einer großen Zahl früherer Einsätze die Geopositionen und den Status aller aktuell im Einzugsbereich eingesetzten Rettungsmittel fortlaufend in die nahe Zukunft zu projizieren, um so die Entscheidung für oder gegen eine Umpositionierung auf einer besseren statistischen Crundlage zu treffen. Eine solche vorausschauende Einsatzmitteldisposition ist in Deutschland bislang nicht realisiert, in anderen Ländern, z.B. den Niederlanden, USA oder Australien dagegen bereits seit Jahren in zahlreichen Regionen routinemäßig im Einsatz.

\section{Disposition der Rettungsmittel}

Ohne leistungsfähige Informationssysteme und leitstellenübergreifende Betrachtung ist heute keine adäquate Disposition von Rettungsmitteln mehr möglich.

\subsection{Smartphone-basierte Ersthelfer-Alarmierung}

Die Reanimationsleitlinien empfehlen neben der T-CPR auch die Alarmierung freiwilliger Ersthelfer über App-basierte Alarmierungssysteme, um die Basisreanimation bis zum Eintreffen des Rettungsdienstes sicherzustellen. Dazu werden nur diejenigen Helfer alarmiert und mittels der integrierten Navigationsfunktion an die Einsatzstelle geleitet, die diesen schneller erreichen können als der Rettungsdienst. Im Juli 2018 waren in 16 Regionen in Deutschland solche Systeme aktiv (Gross et al. 2019). Die weitere Verbreitung dieser Systeme ist sehr dynamisch. Aktuell (Juni 2020) sind nach eigenen Recherchen App-basierte Ersthelferalarmierungen bereits in 45 Regionen mit ca. 13,5 Millionen Einwohnern möglich. Abbildung 3 zeigt ein Beispiel einer solchen App. Die Einrichtung erfolgt i.a. als freiwillige Zusatzleistung von Kommunen, Kreisen und Leitstellen. Der Aufwand zur Klärung des Datenschutzes, der technischen Aspekte, der Finanzierung, der Rechtsstellung und Ausbildung der Helfer sowie des medizinischen QM darf nicht unterschätzt werden. Zudem sind in Deutschland aktuell mindestens 7 unterschiedliche Systeme im Einsatz. Um die größtmögliche Verfügbarkeit von Helfern sicherzustellen, müssen die Leitstellen daher u.U. mehrere Systeme und Schnittstellen zum ELS nutzen. Auch in Dänemark, Teilen der Schweiz, Österreichs oder Italiens sind solche Systeme im Einsatz. Vorliegende Analysen zeigen eine Verkürzung des therapiefreien Intervalls um mehrere Minuten sowie Hinweise auf eine Verbesserung der Überlebensrate (Gross et al. 2019; Auricchio et al. 2019; Stroop et al. 2018). 


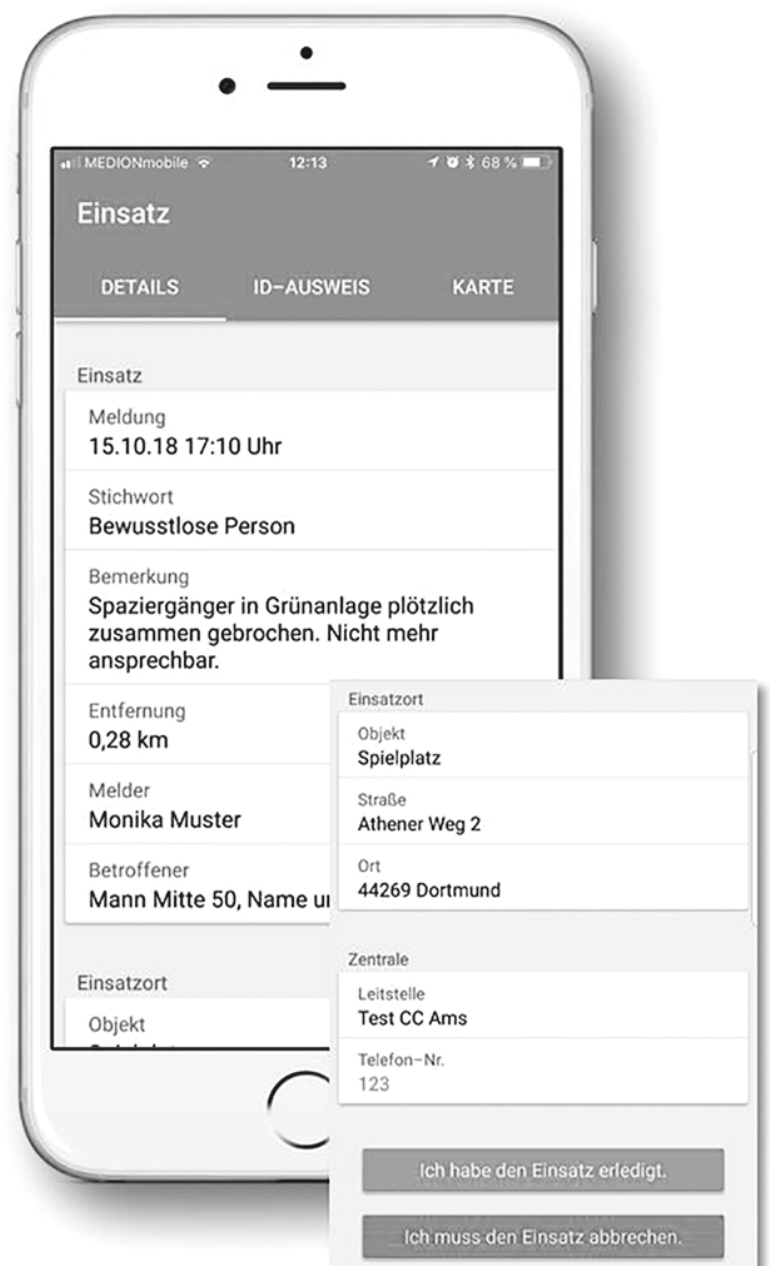

Abb. 3 Mobile Retter App. Dargestellt ist ein Screenshot einer fiktiven Alarmierung (mit freundlicher Genehmigung von Timo Dreier, medgineering $\mathrm{GmbH}$ ).

\section{Das Smartphone als Lebensretter?}

Smarte Apps zur Ersthelferalarmierung haben das Potenzial, das therapiefreie Intervall bei Kreislaufstillstand erheblich zu verkürzen. Die mangelnde Interoperabilität der Systeme erschwert jedoch die breite Umsetzung. 


\subsection{Telemedizinische Unterstützung}

In den USA wurde bereits vor mehreren Jahrzehnten an einzelnen Standorten mit der Funkübermittlung eines EKG (EKG-Telemetrie) von der Einsatzstelle in die Zielklinik begonnen (Uhley 1970). Mit der Einführung der prähospitalen Lyse bei Patienten mit akutem Myokardinfarkt erfuhr diese Technologie dann dort zunehmendes Interesse (Kudenchuk et al. 1998). In Deutschland erfolgte die Entwicklung nur zögerlich. In einer bundesweiten Befragung der Ärztlichen Leiter Rettungsdienst (ÄLRD) war die EKG-Telemetrie 2009 in 43\% der teilnehmenden Rettungsdienstbereiche möglich (Mann et al. 2011). 8 Jahre später lag der Verbreitungsgrad mit 47\% nur wenig höher (Mann et al. 2020). Berücksichtigt man, dass die EKG-Telemetrie 2009 nur in $17 \%$ und 2017 nur in 26\% der Rettungsdienstbereiche aus allen Rettungsmitteln heraus möglich war, relativieren sich die genannten Zahlen weiter. Mehrere Studien belegen den Nutzen der EKG-Telemetrie. So war die Zeit bis zum Beginn der Reperfusion des Infarktgefäßes in einer Metaanalyse um ca . 40\% verkürzt (Brunetti et al. 2017). In Apulien, Italien, überträgt der Rettungsdienst jährlich über $100.000(!)$ EKG. Unter ökonomischen Gesichtspunkten betrachtet war diese Maßnahme mit einem Betrag je Qualitäts-adjustiertem Lebensjahr (QUALY) von nur $2.000 €$ zudem sehr kostengünstig (Brunetti et al. 2014).

Seit einigen Jahren können vom Notfallort aus, eine entsprechende technische Ausstattung vorausgesetzt, auch kontinuierlich sämtliche Vitaldaten (Blutdruck, Sauerstoffsättigung, etc.) übertragen werden. Die nunmehr mögliche Zusammenschau einer Vielzahl an Parametern erleichtert die Einschätzung des Patientenzustands durch die Zielklinik und bildet die Grundlage für eine telemedizinische Unterstützung im Einsatz. Federführend beim Aufbau eines „Telenotarztsystems“ waren Aachen und einige Nachbarkreise, wo dieser Dienst mittlerweile regulärer Teil des Rettungsdienstes ist (s. Kap. I.10). Neben dem Vitaldatenstreaming wird dazu auch eine Telefonverbindung genutzt, die situationsabhängig durch die Übertragung von Standbildern und Videostreaming ergänzt wird. Die telenotärztliche Unterstützung kann v.a. dazu beitragen, die physische Präsenz eines Notarztes bei der großen Zahl an Patienten ohne reale Vitalbedrohung zu reduzieren. In Baden-Württemberg z.B. machen solche Patienten ca. 30\% aller Notarzteinsätze aus (SQR-BW 2019). Im Vergleich zum herkömmlichen Notarzt ist die Einschaltung des Telenotarztes ohne Zeitverzug möglich, seine zeitliche Bindung ist erheblich geringer als bei konventionellen Notarzteinsätzen, und er kann auch Parallelanforderungen bedienen.

Telemedizin trägt die Expertise notfallmedizinischer Zentren in die Regionen.

In Fall-Kontroll-Studien aus Aachen war die telenotärztlich supervidierte, von Rettungsfachpersonal vor Ort durchgeführte Behandlung bei hypertensiver 
Entgleisung oder akutem Schmerzsyndrom mit einer Behandlung durch den am Notfallort physisch präsenten Notarzt qualitativ vergleichbar (Brokmann et al. 2017; Lenssen et al. 2017). Aktuell wird eine vergleichende prospektiv randomisierte Studie aus Aachen zur Frage Telenotarzt vs. „normaler“ Notarzt ausgewertet. Ihre Ergebnisse werden erhebliche Auswirkungen hinsichtlich der weiteren Verbreitung von Telenotarztdiensten haben. Neben der o.g. Region haben aktuell lediglich die Kreise Vorpommern-Greifswald, Straubing und Main-Kinzig-Kreis den Telenotarzt als Pilotvorhaben implementiert. Nordrhein-Westfalen und Bayern haben allerdings bereits beschlossen, den Telenotarztdienst flächendeckend auszubauen.

\subsection{Echtzeitkapazitätsnachweise der Kliniken}

Eine „Schlüsselstelle“ der Rettungskette ist die Wahl einer aufnahmebereiten, fachlich geeigneten Zielklinik. Ohne IT-Unterstützung sind oftmals mehrere Telefonate notwendig, bis geklärt ist, ob eine Aufnahme erfolgen kann. Der Zeitverlust erreicht nicht selten 15 Minuten, was 25\% des im Eckpunktepapier genannten maximalen Prähospitalintervalls entspricht! Zudem binden diese Aktivitäten die Aufmerksamkeit des Notfallteams. Die Lösung besteht in Echtzeitdatenbanken, welche die für den Rettungsdienst relevanten Klinikkapazitäten in einer ständig aktualisierten, online zugänglichen Datenbank darstellen und daraus ggf. direkt eine Patientenzuweisung an eine bestimmte Klinik generieren können.

\section{Der „Telefonmarathon“ zur Zuweisung von Notfallpatienen muss ein Ende} haben!

Dabei erlauben Filter die Differenzierung nach Alter, Typ der Versorgung (Notaufnahme, Intensivstation, Normalstation), Fachrichtung etc. Die Datenbankabfrage erfolgt in der Regel durch die Leitstelle. Wo mobile Tablet-PCs zur Einsatzdokumentation genutzt werden, bietet sich die direkte Abfrage durch das Notfallteam vor Ort an. Diese Systeme sind nicht nur im Individualnotfall, sondern auch beim MANV oder in Infektionslagen, wie aktuell COVID-19, wichtig, wo sie maßgeblich zu einer besseren Lagedarstellung und Patientenverteilung beitragen können. Mittlerweile verfügen Berlin², Brandenburg, Bremen, Hessen, Nordrhein-Westfalen, Rheinland-Pfalz und das Saarland über landesweite Systeme. In Bayern, Baden-Württemberg, Niedersachsen und Schleswig-Holstein sind Teile des Landes damit ausgestattet. Unterschiedliche Anbieter, Philosophien, Funktionsumfänge und Nomenklaturen erschweren allerdings die überregionale Nutzung.

2 Zwar nutzt die Berliner Integrierte Leitstelle einen Echtzeitkapazitätsnachweis. Der Implementierungsgrad in den Kliniken variiert allerdings. 


\subsection{Einsatzdokumentation und Qualitätsmanagement}

Die papierbasierte Einsatzdokumentation weist eine Reihe von Nachteilen auf. Die Lesbarkeit ist eingeschränkt und die Dokumentationsqualität häufig ungenügend (Bergrath et al. 2011). Die Auswertung ist generell, v.a. in größeren Rettungsdienstbereichen, mit einem enormen Aufwand verbunden, und ein wirksames QM wird durch unvollständige oder unplausible Einsatzdaten massiv erschwert. Baden-Württemberg initiierte daher schon vor vielen Jahren eine elektronische Nachbearbeitung der papierbasierten Notarztprotokolle, wodurch erstmals eine landesweite Auswertung möglich wurde (Messelken et al. 2010). In einer 2011 publizierten bundesweiten Umfrage unter Ärztlichen Leitern Rettungsdienst ( $̈$ LRD) erfolgte die Einsatzdokumentation der RTW-Besatzungen bei $88 \%$ der Teilnehmer noch papierbasiert, davon bei einem kleinen Anteil mit digitaler Weiterverarbeitung (Video-Pen, Belegleser, Scanner). 78\% der ÄLRD gaben allerdings an, ein digitales System einführen zu wollen (Mann et al. 2011). 2017 nutzten 59\% der teilnehmenden Rettungsdienstbereiche allerdings immer noch Papierprotokolle, während 37\% die Einsatzdaten digital (i.d.R. mittels Tablet-PCs) erfassten (Mann et al. 2020).

Ein großer Vorteil der digitalen Einsatzdatenerfassung besteht darin, dass die Vitalparameter und das Ruhe-EKG von angebundenen Medizingeräten drahtlos automatisch in die Dokumentation übernommen werden können. Fotos von der Unfallstelle oder dem Medikationsplan lassen sich der Dokumentation ohne Aufwand hinzufügen. Im Rendezvous-Einsatz können Protokolle mit geringem Aufwand zwischen RTW und NEF elektronisch übergeben werden. Die Systeme lassen sich auch zur Einsatzunterstützung nutzen, z.B. durch interaktive Einblendung von Leitlinien, Aufruf von Arzneimitteldatenbanken oder Übersetzungshilfen. Zudem können bereits im Einsatz erste Informationen digital an Zielkliniken übermittelt werden, z.B. Status der Vitalfunktionen, Arbeitsdiagnose, Infektionsstatus sowie voraussichtliche Eintreffzeit. Dies kann wesentlich dazu beitragen, Informations- und Zeitverluste zu minimieren. Sofern eine Schnittstelle zum Klinikinformationssystem besteht, kann auch das komplette Einsatzprotokoll in die Klinik übermittelt werden. Dies war 2017 bereits in 27\% der Rettungsdienstbereiche technisch möglich (Mann et al. 2020). Mithilfe der Einsatznummer des Rettungsdienstes als gemeinsamem Identifikationsmerkmal kann die Klinik ihrerseits für das medizinische QM relevante Parameter, wie Entlassdiagnose oder Entlassart, an den Rettungsdienstträger zurückspiegeln. Schließlich können auch die Einsatzvor- und -nachbearbeitung, z.B. Dokumentation von Gerätecheck und Materialverbrauch sowie Fakturierung erheblich erleichtert werden.

Diesen Vorzügen stehen allerdings auch Herausforderungen gegenüber. So sind über einen Nutzungszeitraum von 5 Jahren Kosten in Höhe von mehreren tausend Euro zu veranschlagen (Tablet-PC inkl. Ladehalterung und ggf. mobiler Drucker, Lizenzen für Erfassungssoftware, Schnittstellen, und Auswertesoftware, Mobilfunkgebühren). Je nach Umfang zusätzlicher Schnittstellen 
Tab. 1 Gegenüberstellung papierbasierter und digitaler Einsatzdokumentation

\begin{tabular}{|c|c|c|}
\hline \multicolumn{2}{|r|}{ Papierdokumentation } & Digitale Dokumentation \\
\hline $\begin{array}{l}\text { Vor- } \\
\text { teile }\end{array}$ & $\begin{array}{l}\text { einfache Beschaffung } \\
\text { einfache Einarbeitung } \\
\text { ausfallsicher } \\
\text { - sehr niedrige Kosten }\end{array}$ & $\begin{array}{l}\text { höhere Dokumentationsqualität } \\
\text { automatische Einbettung weiterer Einsatzbezogener } \\
\text { Dokumente } \\
\text { n } \text { automatische Datenübernahme aus Medizingeräten } \\
\text { nutzung auch als Informationssystem } \\
\text { Unterstützung der Einsatzvor- und Nachbereitung } \\
\text { Datenversand an Zielkliniken } \\
\text { umfassende Datenbasis für das medizinische QM }\end{array}$ \\
\hline $\begin{array}{l}\text { Nach- } \\
\text { teile }\end{array}$ & $\begin{array}{l}\text { schlechte Lesbarkeit } \\
\text { oft ungenügende } \\
\text { Dokumentationsqualität } \\
\text { geringe Eignung für das } \\
\text { medizinische QM } \\
\text { nicht nutzbar für Einsatzvor- } \\
\text { und Nachbereitung }\end{array}$ & $\begin{array}{l}\text { aufwändige Beschaffung } \\
\text { höherer Bedienungsaufwand (abhängig vom } \\
\text { System) } \\
\text { abhängig von Akkuleistung und ggf. drahtlosen } \\
\text { Verbindungen } \\
\text { hohe Kosten }\end{array}$ \\
\hline
\end{tabular}

können diese über den genannten Zeitraum nochmals einen vierstelligen Betrag erfordern. Für den Umstellungsprozess auf die digitale Dokumentation (Anforderungsanalyse, Beschaffung, Etablierung des Rechte- und Rollensystems, Mitarbeiterschulung, Ertüchtigung der Schnittstellen, Stammdatenpflege) müssen ausreichend personelle Ressourcen verfügbar sein. Zudem müssen bei Ausfällen Ersatzgeräte und ggf. auch Papierprotokolle als letzte Redundanzebene vorgehalten werden (s. Tab. 1).

Aktuell wird in Baden-Württemberg, Bayern, Rheinland-Pfalz und Hamburg landesweit digital dokumentiert. In den meisten anderen Bundesländern sind solche Systeme regional im Einsatz. Insgesamt dürften ca. 70\% der Rettungsdienstbereiche digitale Dokumentationssysteme nutzen. In Österreich sind drei Bundesländer flächendeckend ausgerüstet, in der Schweiz nur einzelne Standorte, z.B. Zürich.

\subsection{Rahmenbedingungen für die erfolgreiche Implementierung digitaler Technologien}

Je weiter die Digitalisierung im Rettungswesen voranschreitet, desto wichtiger wird die Absicherung gegen intentionelle (unbefugter Zugang, Manipulation und Diebstahl von Daten, Sabotage) oder akzidentelle Störungen (v.a. Naturgewalten, Stromausfall). Die dafür notwendige Resilienz kann nur durch eine Kombination technischer und organisatorischer Maßnahmen und eine geeignete Aus- und Fortbildung der Mitarbeiter erzielt werden. In einer systematischen Risikoanalyse sollten nicht nur Einzelfaktoren, sondern auch Kombinationen von Risiken betrachtet und entsprechende Gegenmaßnahmen 
(z.B. aktuelle Verschlüsselungstechnologien, biometrische Zugangskontrollen, redundante Zuleitungen, Ersatzleitstellen) umgesetzt werden. In einem dritten Schritt ist die tatsächliche Resilienz durch regelmäßige Übungen zu überprüfen. Eine wichtige Grundlage für die notwendige Absicherung bilden die vom Bundesamt für Sicherheit in der Informationstechnik empfohlenen BSI-Standards 200-1, 200-2 und 200-3.

Die mit der Datennutzung zusammenhängenden Rechtsfragen müssen frühzeitig, bereits während der Anforderungsanalyse der zu beschaffenden Systeme, sorgfältig betrachtet werden.

Für die Beschaffung und den Unterhalt der IT-Systeme sind ausreichend Finanzmittel sowie personelle Ressourcen für das Projektmanagement und die Systemadministration bereit zu stellen.

Die Digitalisierung zentraler Arbeitsprozesse muss bei der Ausbildung stärker berücksichtigt werden. Bezüglich der Qualifikationsanforderungen an das Leitstellenpersonal ist die Digitalisierung ein zentraler Punkt eines Grundsatzpapiers der Leiter der Berufsfeuerwehren (AGBF) im Deutschen Städtetag und des Deutschen Feuerwehr-Verbands (Deutscher Städtetag 2019).

Digitale Anwendungen im Rettungsdienst benötigen leistungsfähige Mobilfunknetze. Daher müssen die im ländlichen Bereich bestehenden Lücken, v.a. im LTE-Netz, rasch geschlossen werden.

Die Nutzung von Verfahren der Künstlichen Intelligenz als sog. disruptive Technologie wird das Arbeitsumfeld im Rettungsdienst mittelfristig maßgeblich beeinflussen. Wichtige Aspekte sind, wie KI-unterstützte Entscheidungen angemessen dokumentiert und nachvollzogen werden können, wie sie haftungsrechtlich zu betrachten sind, und wie Mitarbeiter am besten auf die Technologie vorbereitet werden.

Take home messages: Erfolgsfaktoren bei der Einführung digitaler Technologien im Rettungsdienst

- systematische Risikoanalyse

- Absicherung der Systeme gegen Störungen

- Anwendung der BSI-Standards zur Sicherheit in der Informationstechnologie

- frühzeitige Klärung von Rechtsfragen zur Datennutzung

- Qualifizierung der Mitarbeiter

- ausreichende Personalressourcen zur Projekt- und Systembetreuung

- Ausbau des Mobilfunknetzes im ländlichen Raum

- Vorbereitung auf disruptive Technologien 


\section{Literatur}

Arbeitsgemeinschaft der Leiter der Berufsfeuerwehren (AGBF) im Deutschen Städtetag, Deutscher Feuerwehrverband (2019) Grundsatzpapier (2019): Qualifikationsanforderungen für Leitstellenpersonal Integrierter Leitstellen (ILS) für Feuerwehr, Rettungsdienst und Katastrophenschutz. URL: http://www.agbf.de/ downloads-ak-integrierte-leitstelle/category/53-fa-ils-oeffentlich-grundsatzpapier.html?download=323: qualifikationsanforderungen-fuer-leitstellenpersonal.pdf (abgerufen am 12.08.2020)

Auricchio A, Gianquintieri L, Burkart R, Benvenuti C, Muschietti S, Peluso S, Mira A, Mocetti T, Caputo ML (2019) Real-life time and distance covered by lay first responders alerted by means of smartphone-application: Implications for early initiation of cardiopulmonary resuscitation and access to automatic external defibrillators. Resuscitation 141, 182-187

Baumann A, Sellin S, Breckwoldt I (2009) Standardisierte Notruf-Abfragesysteme für die Leitstelle. NotfallmedUp2date 4, 261-275

Bergrath S, Skorining M, Rörtgen M, Beckers SK, Brokmann IC, Mutscher C, Rossaint R (2011) Is paper-based documentation in an emergency medical service adequate for retrospective scientific analysis? An evaluation of a physician-run service. Emerg Med I 28, 320-324

Blomberg SN, Folke F, Ersbøøll AK, Christensen HC, Torp-Pedersen C, Sayre MR, Counts CR, Lippert FK (2019) Machine learning as a supportive tool to recognize cardiac arrest in emergency calls. Resuscitation 138, 322-329

Böttiger BW, Becker LB, Kern KB, Lippert F, Lockey A, Ristagno G, Semeraro F, Wingen S (2020) BIG FIVE strategies für survival following out-of-hospital cardiac arrest. Eur | Anaesthesiol 37, 1-4

Brokmann IC, Roisssant R, Müller M, Fitzner C, Villa L, Beckers SK, Bergrath S (2017) Blood pressure management and guideline adherence in hypertensive emergencies and urgencies: A comparison between telemedically supported and conventional out-of-hospital care. I Clin Hyptens (Greenwich) 19, 704-712

Brunetti ND, De Gennaro L, Correale M, Santoro F, Caldarola P, Gaglione A, Di Biase M (2017) Pre-hospital Electrocardiogram Triage With Telemedicine Near Halves Time to Treatment in STEMI: A Meta-Analysis and Meta-Regression Analysis of Non-Randomized Studies. Int I Cardiol 232, 5-11

Brunetti ND, Dellegrottaglie G, Lopriore C, Di Guiseppe G, De Gennaro L, Lanzone S, Di Biase M (2014) Prehospital Telemedicine Electrocardiogram Triage for a Regional Public Emergency Medical Service: Is It Worth It? A Preliminary Cost Analysis. Clin Cardiol 37, 140-145

Buck BH, Starkman S, Eckstein M, Kidwell CS, Haines I, Huang R, Colby D, Saver JL (2009) Dispatcher Recognition of Stroke Using the National Academy Medical Priority Dispatch System. Stroke 40, 2027-2030

Bundesgesundheitsministerium (2020) Referentenentwurf zur Reform der Notfallversorgung. URL: https:// www.bundesgesundheitsministerium.de/fileadmin/Dateien/3_Downloads/Gesetze_und_Verordnungen/GuV/N/Referentenentwurf_zur_Reform_der_Notfallversorgung.pdf (abgerufen am 12.08.2020)

Deutscher Städtetag (2019) Digitalisierung im Brand-, Katastrophenschutz und Rettungswesen. Diskussionspapier des Deutschen Städtetages. Hamm. URL: http://www.staedtetag.de/imperia/md/content/dst/veroeffentlichungen/mat/digitalisierung-brand-katastrophenschutz-rettungswesen-diskussionspapier-2019. pdf (abgerufen am 12.08.2020)

Deakin CD, Alasaad M, King P, Thompson F (2009) Is Ambulance Telephone Triage Using Advanced Medical Priority Dispatch Protocols Able to Identify Patients With Acute Stroke Correctly? Emerg Med I 26, 442-445

Deakin CD, England S, Diffey D (2017) Ambulance Telephone Triage Using 'NHS Pathways' to Identify Adult Cardiac Arrest. Heart 103, 738-744

Ellensen EN, Naess H, Wisborg T, Hunskaar S, Zakariassen E (2018) Stroke identification by criteria based dispatch - a register based study. Acta Anaesthesiol Scand 62, 105-115

European Commission, Electronic Communications Networks and Services, Communications Committee, Working Document (2020) Implementation of the single European emergency number 112 - Results of the thirteenth data-gathering round. Brüssel. URL: https://ec.europa.eu/newsroom/dae/document. cfm?doc_id=64510 (abgerufen am 12.08.2020)

Fischer M, Seewald S, Gräsner J-T, Jakisch B, Bohn A, Jantzen T, Brenner S, Bein B, Wnent I (2018) Außerklinische Reanimationen im Deutschen Reanimationsregister - eine Übersicht der Jahre 2014 bis 2017. Anästh Intensivmed 59, 679-682 
Fischer M, Kehrberger E, Marung H, Moecke H, Prückner S, Trentzsch H, Urban B, Fachexperten der Eckpunktepapier Konsensus-Gruppe (2016) Eckpunktepapier 2016 zur notfallmedizinischen Versorgung der Bevölkerung in der Prähospitalphase und in der Klinik. Notfall Rettungsmed 19, 387-395

Gebert A, Van Lengen R, Moucha C, Putz W, Schmitt M, Schröder M (2015) SUSI TD - Sicherheit und Unterstützung für Senioren durch Integration von Technik und Dienstleistung. S. 77-82. In: 8. Deutscher AAL-Kongress. Gemeinsam mit: Zukunftslebensräume- Bauen, Wohnen und Gesundheit im Wandel. Ambient Assisted Living Deutschland (AAL). VDE Verband der Elektrotechnik Elektronik Informationstechnik e.V. - Berlin: VDE-VERLAG.

Gräsner IT, Wnent I, Herlitz I, Perkins GD, Lefering R, Tjekmeland I, Koster RW, Masterson S, Rossell-Ortiz F, Maurer H, Böttiger BW, Moertl M, Mols P, Alihodžić H, Hadžibegović I, loannides M, Truhlár A,Wissenberg M, Salo A, Escutnaire J, Nikolaou N, Nagy E, Steinn)onsson B, Writht P, Semeraro F, Clarens C, Beesems S, Cebula G, Correia VH, Cimpoesu D, Raffey V, Trenkler S, Markota A, Strömsöe A, Burkart R, Booth S, Bossaert L (2020) Survival after out-of-hospital cardiac arrest in Europe - Results of the EuReCa TWO study. Resuscitation 148, 218-226

Gross B, Schanderl F, Staedt N, Elsner C (2019) App-basierte Systeme zur Ersthelferalarmierung. Stand der Verbreitung in Deutschland. Evidenz und Herausforderungen bei der Einführung. Notfall Rettungsmed 22, 483-491

Heward A, Damiani M, Hartley-Sharpe C (2004) Does the use of the Advanced Medical Priority Dispatch System affect cardiac arrest detection? Emerg Med | 21, 115-118

Höfer C, Lefering R, Sektion Intensiv- \& Notfallmedizin, Schwerverletztenversorgung der Deutschen Gesellschaft für Unfallchirurgie e.V. AUC- Akademie der Unfallchirurgie GmbH (2018). Traumaregister DGU. Jahresbericht 2018. URL: http://www.traumaregister-dgu.de/fileadmin/user_upload/traumaregister-dgu.de/ docs/Downloads/TR-DGU-Jahresbericht_2018.pdf (abgerufen am 12.08.2020)

Institut für Notfallmedizin und Medizinmanagement, Klinikum der Universität München (2005) Trend- und Strukturanalyse des Rettungsdienstes in Bayern (TRUST-Studie). 1999-2004. Abschlussbericht. Band I: Ergebnisse für das Rettungswesen in Bayern. München, August 2005. URL: https://www.innenministerium. bayern.de/assets/stmi/sus/rettungswesen/id3_27_veroeffentlichungen_trust2004_band1_20130222. pdf (abgerufen am 12.08.2020)

Kudenchuk, PI, Maynard C, Cobb LA, Wirkus M, Martin IS, Kennedy IW, Weaver WD (1998) Utility of the Prehospital Electrocardiogram in Diagnosing Acute Coronary Syndromes: The Myocardial Infarction Triage and Intervention (MITI) Project. I Am Coll Cardiol 32, 17-27

Lee SY, Song KJ, Shin SD, Hong KJ, Kim TH (2020) Comparison of the effects of audio-instructed and videoinstructed dispatcher-assisted cardiopulmonary resuscitation on resuscitation outcomes after out-of-hospital cardiac arrest. Resuscitation 147, 12-20

Lenssen N, Krokauer A, Beckers SK, Rossaint R, Hirsch F, Brokmann IC, Bergrath S (2017) Quality of analgesia in physician operated telemedical prehospital emergency care is comparable to physician-based prehospital care - a retrospective longitudinal study. Sci Rep 7, 1536

Luiz T, Marung H, Pollach G, Hackstein A (2019) Implementierungsgrad der strukturierten Notrufabfrage in deutschen Leitstellen und Auswirkungen ihrer Einführung. Anaesthesist 68, 282-293

Mann V, Zajonz TS, Mann ST, Müller M, Edeler B, Schneck E, Sander M, Brenk F (2020) Moderne Techniken der präklinischen Notfallmedizin in Deutschland. Fünf-Jahres-Follow-Up einer Erhebung unter den ärztlichen Leitern Rettungsdienst. Anästh Intensivmed 61, 1-14

Mann V, Brammen D, Brenk F, Euler M, Messelken M, Röhrig R (2011) Innovative Techniken in der präklinischen Notfallmedizin in Deutschland. Eine Online-Erhebung unter den Ärztlichen Leitern Rettungsdienst. Anästhesiol Intensivmed 52, 824-833

Messelken M, Kehrberger E, Dirks B, Fischer M (2010) Notärztliche Versorgungsqualität in Baden-Württemberg: Realität im Längsschnitt von vier Jahren. Dtsch Arztebl Int 107, 523-530

Palma E, Antonaci D, Coli A, Cicolini G (2014) Analysis of emergency medical services triage and dispatch errors by registered nurses in Italy. I Emerg Nurs 40, 476-483

Peleg K, Pliskin IS (2004) A Geographic Information System Simulation Model of EMS: Reducing Ambulance Response Time. Am | Emerg Med 22, 164-170 
Sanson G, Verduno I, Zambon M, Trevi R, Caggegi GD, Di Bartolomeo S, Antonaglia V (2016). Emergency medical service treated out- of-hospital cardiac arrest: Identification of weak links in the chain-of-survival through an epidemiological study. Eur I Cardiovasc Nurs 15, 328-333

Soar I, Maconochie I, Wyckhoff MH, ILCOR Task Force (2019) 2019 International Consensus on cardiopulmonary resuscitation and emergency cardiovascular care science with treatment recommendations. Resuscitation $145,95-150$

Sellin S, Baumann A, Breckwoldt I (2009) Einfluss eines standardisierten Notrufabfrage-Protokolls (SNAP) auf die Qualität von Notrufgesprächen bei Herz-Kreislauf-Stillstand. Notfall Rettungsmed 12 [Suppl 1], 1-17

SQR BW, Stelle zur trägerübergreifenden Qualitätssicherung im Rettungsdienst Baden-Württemberg (SQR BW) (2018) Qualitätsbericht 2018. URL: https://www.sqrbw.de/adbimage/1428/asset-original//qualitaetsbericht-2018.pdf (abgerufen am 12.08.2020)

Stipulante S, Tubes R, El Fassi M, Donneau A-F, Van Troyen B, Hartstein G, D’Orio V, Ghuysen A (2014) Implementation of the ALERT algorithm, a new dispatcher-assisted telephone cardiopulmonary resuscitation protocol, in non-Advanced Medical Priority Dispatch System (AMPDS) Emergency Medical Services. Resuscitation $85,177-181$

Stroop R, Hensel M, Schnettker AT, Strickmann B, Kuhlbusch T, Kerner T (2018) Smartphone-basierte Ersthelfer-Alarmierung verkürzt das reanimationsfreie Intervall. Eine Machbarkeitsstudie zur Verbesserung der präklinischen Reanimation. Anästh Intensivmed 59, 58-67

Uhley HN (1970) Electrocardiographic telemetry from ambulances. A practical approach to mobile coronary care units. Am Heart / 80, 838-842

Shimamoto T, Iwami T, Kitamura T, Nishiyama C, Sakai T, Nishiuchi T, Hayashi Y, Kawamura T, Utstein Osaka Project (2015) Dispatcher instruction of chest compression-only CPR increases actual provision of bystander CPR. Resuscitation 96, 9-15

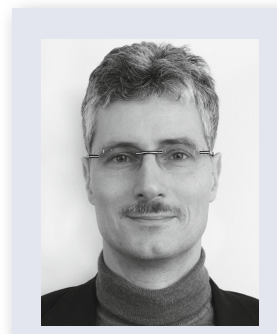

PD Dr. med. Thomas Luiz

Thomas Luiz ist Project Manager im Programm „Digital Healthcare“ am Fraunhofer IESE in Kaiserslautern. Schwerpunkte der aktuellen wissenschaftlichen Tätigkeit des Anästhesisten und aktiven Notfallmediziners sind Struktur- und Prozessanalysen in der Notfallmedizin, Projektmanagement bei der Einführung digitaler Technologien im Rettungsdienst und die Erforschung virtueller Lernumgebungen in der medizinischen Aus-, Fort- und Weiterbildung. 